\title{
Increased risk of low bone mineral density in patients with non-alcoholic fatty liver disease: a cohort study
}

\section{Zhe Shen ${ }^{1, *}$, Li Cen ${ }^{1, *}$, Xufeng Chen ${ }^{1,2}$, Jiaqi Pan ${ }^{1}$, Youming Li', Weixing Chen ${ }^{1}$ and Chaohui $\mathbf{Y u}^{1}$}

'Department of Gastroenterology, The First Affiliated Hospital, College of Medicine, Zhejiang University, Hangzhou, China and ${ }^{2}$ Department of Emergency, Zhejiang Hospital, Hangzhou, China

*(Z Shen and L Cen contributed equally to this work)
Correspondence should be addressed to W Chen or C Yu Email 21718072@zju.edu.cn or zyyyych@zju.edu.cn

\begin{abstract}
Background: Non-alcoholic fatty liver disease (NAFLD) has been suggested to be a risk factor associated with low BMD (bone mineral density) in several cross-sectional studies. The present longitudinal cohort study aims to explore the effect of NAFLD and its severity on low BMD.

Methods: Between January 2013 and August 2018, individuals who participated in annual comprehensive health examinations were included. BMD was presented using dual-energy X-ray absorptiometry (DXA). These subjects were diagnosed with fatty liver by ultrasound detection.

Results: A total of 1720 subjects were included (1064 subjects with normal BMD and 656 subjects with low BMD) at baseline. Among the 1064 participants with normal BMD at baseline, 399 participants developed low BMD. The multivariable-adjusted hazard ratio for incident low BMD comparing the NAFLD group vs the non-NAFLD group was $2.24(1.18,2.81)$. Increased non-invasive fibrosis markers of NAFLD were positively associated with an increased incidence of low BMD in a graded manner. In addition, obese subjects and women with NAFLD at baseline are more likely to develop low BMD.

Conclusion: NAFLD and its severity were independently associated with an increased incidence of low BMD. Obesity and female gender are risk factors associated with low BMD. Our findings indicated NAFLD can be a significant contributor to low BMD pathogenesis, requiring further studies to elucidate the potential mechanisms.
\end{abstract}

\section{Introduction}

Osteoporosis is a common systemic skeletal disease characterized by reduced bone strength and could predispose to an increased fracture risk. The integration of bone mineral density (BMD) and bone quality could reflect the bone strength. Bone quality is hard to assess on a population basis, while BMD is a well-defined predictor of fracture risk and is easily measurable (1). Global death and disability-adjusted life years attributable to low BMD have almost doubled over the past two decades, and approximately one-third of fall-related deaths are attributable to low BMD (2). Hence, identifying risk factors with early preventive interventions are crucial in the management of low BMD.

Non-alcoholic fatty liver disease (NAFLD), which is one of the most common liver diseases, is associated with heavy health and socioeconomic burdens worldwide. NAFLD is defined as excessive fat infiltration to the liver in the absence of significant alcohol intake or secondary causes. At present, the population prevalence of NAFLD in Asia is approximately 25\%, which is similar to many

Published by Bioscientifica Ltd. 
Western countries (3). Furthermore, the prevalence and development of NAFLD are closely associated with sedentary lifestyles, and the increasing incidence of metabolic disorders, such as obesity, type- 2 diabetes mellitus, dyslipidemia and hypertension (4).

Both liver and bone are active endocrine organs that exert several metabolic effects $(5,6)$. Recently, increasing evidence suggests a potential link between NAFLD and low $\operatorname{BMD}(7,8,9,10)$. Several studies have demonstrated that patients with NAFLD are likely to have low BMD and increased risk of osteoporotic fractures, and the related mechanism has been considered to be complex and unknown (7). Few studies demonstrated that the presence of significant liver fibrosis assessed via transient elastography (TE) was independently associated with low BMD in NAFLDs (11). Limited large-scale longitudinal studies have elucidated the association between low BMD and NAFLD. Moreover, the mechanism behind this is not fully understood, and chronic inflammation, insulin resistance (IR) and vitamin D deficiency appear to provide potential links (12).

Therefore, the present longitudinal cohort study was conducted to determine whether the NAFLD and its severity, based on non-invasive fibrosis markers, is associated with the development of low BMD compared with no NAFLD.

\section{Methods}

\section{Study design and subjects}

The present longitudinal cohort study aimed to investigate the impact of NAFLD on the development of low BMD. All individuals participated in the annual comprehensive health examinations at the Health Center of the First Affiliated Hospital, College of Medicine, Zhejiang University between January 1, 2013, and August 31, 2018. Concrete flow diagram for the selection of study subjects was presented in Fig. 1 and Supplementary material (see section on supplementary materials given at the end of this article).

\section{Measurements of clinical variables and biochemical data}

All variables were assessed at each visit at the same place. Data on demographic characteristics and personal history were collected through a self-administered health questionnaire. Besides detailed physical measurements,

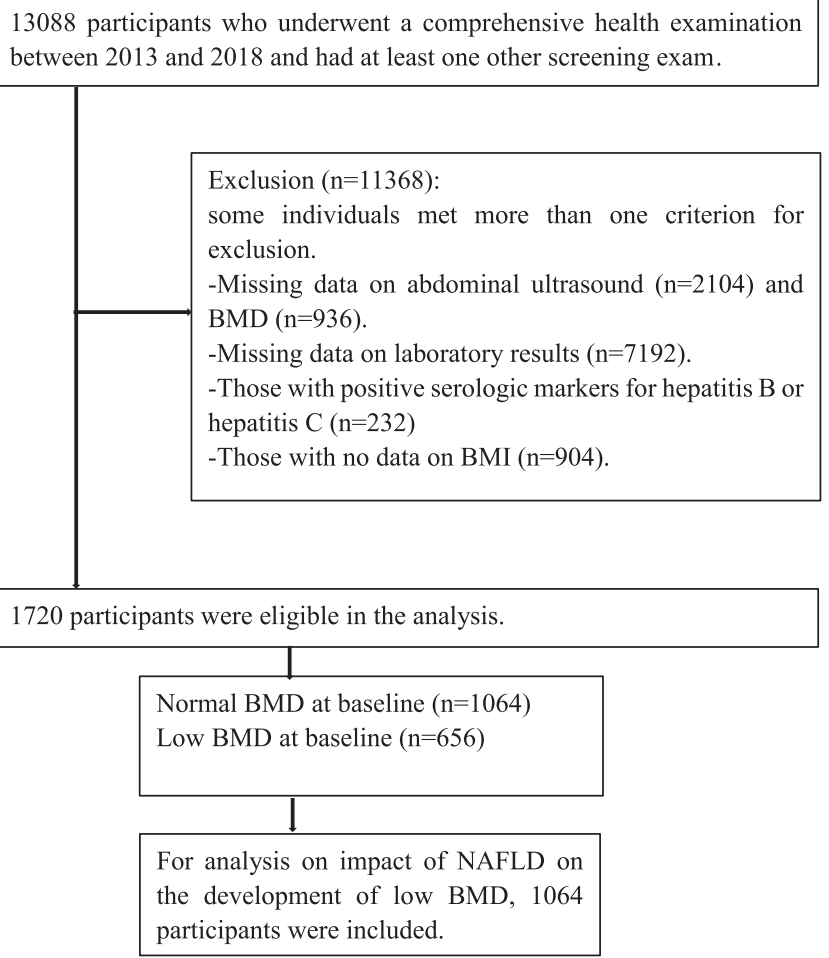

\section{Figure 1}

Flow diagram for the selection of study subjects.

DXA scans, ultrasound, and serum biochemical parameters were measured by trained staff during the health examinations. Supplemental material includes concrete measuring methods and diagnostic criteria.

\section{Measurement and definition of low BMD}

Low BMD in the present study included osteoporosis and osteopenia, according to WHO (13) . Specifically, osteoporosis is defined as a T-score of less than or equal to -2.5 on the DXA of at least one of the three skeletal sites (lumbar spine, femur neck, or total hip), while osteopenia is defined as a T-score between -1 and -2.5 at any one of the three sites. In our study, osteoporosis and osteopenia were defined with a T-score of two skeletal sites (lumbar spine and total hip). Since only a small amount of participants were identified with osteoporosis, we combined osteoporosis and osteopenia into one group called low BMD group.

\section{Definition of NAFLD and its severity}

NAFLD is defined as the presence of fatty liver in the absence of excessive alcohol use or other identifiable 
cause. An ultrasonographic diagnosis of fatty liver was determined based on characteristic echo patterns, according to conventional criteria (14).

To assess the risk of severe NAFLD, two non-invasive indices of liver fibrosis were used: fibrosis-4 (FIB-4) and aspartate transaminase-to-platelet ratio index (APRI) $(15,16)$. FIB-4 was calculated according to the following formula: $\quad$ FIB- $4=($ age $\quad($ years $) \times$ AST $\quad(\mathrm{U} / \mathrm{L})) /($ platelet count $\left(\times 10^{9} / \mathrm{L}\right) \times$ ALT $\left.(\mathrm{U} / \mathrm{L})^{1 / 2}\right)$. Cut-offs were used to define low (FIB-4 <1.30), intermediate, and high (FIB-4 $\geq 2.67$ ) probabilities of advanced fibrosis (15). The APRI was calculated by the following formula: APRI $=100 \times$ (AST/upper limit of normal)/platelet count $\left(\times 10^{9} / \mathrm{L}\right)$. Cutoffs for low and high probability of advanced fibrosis were 0.5 and 1.5 respectively (16). Since only a small amount of participants were identified as having both NAFLD and a high probability of advanced fibrosis, we combined the intermediate and high fibrosis score groups.

\section{Outcome assessment}

All variables were assessed at each visit. The incidence of low BMD cohort was defined as participants who were free of low BMD at baseline, but developed low BMD by the end of follow-up, and considered to be incidental cases of low BMD. For incidental cases of low BMD, the follow-up time was calculated as the difference between baseline and the examination when the incidence of low BMD was identified. For participants who did not develop low $\mathrm{BMD}$, the follow-up time was censored at the last known follow-up examination.

Considering gender is closely associated with the prevalence of NAFLD and low BMD, we did separate statistical analyses for men and women. Since NAFLD is closely associated with obesity (BMI $\geq 25 \mathrm{~kg} / \mathrm{m}^{2}$ ), and simple adjustment for BMI may not be enough to control for the effects of obesity, we performed subgroup analysis based on the presence of obesity to evaluate the association between NAFLD and low BMD in non-obese and obese individuals in men and women.

To reduce several potential variables, we included those in the multivariable models. Model 1 was adjusted for age, gender and BMI; model 2 was adjusted for model 1 plus serum calcium $(\mathrm{Ca})$ and smoking status. $P<0.05$ (two-tailed test) was considered statistically significant.

\section{Propensity score matching}

For such a longitudinal cohort study, selection bias is always a concern. To better assess the role of NAFLD in the development of low BMD, a 1:1 nearest-neighbor propensity score matching (PSM) with a caliper width of 0.02 was performed. Chi-squared tests were used to examine the covariate balance before and after PSM. PSM was performed between the NAFLD and non-NAFLD groups. Concrete method is included in the supplemental material.

\section{Statistical analysis}

Statistical analyses were performed using the SPSS software package, version 22.0 for Windows (SPSS Inc.). All continuous variables are presented as mean \pm s.D., and categorical variables are expressed as frequencies with percentages. ANOVA was conducted for continuous variables, while chi-square tests were conducted for categorical variables. Cumulative event rates for the incidence of low BMD were estimated using Kaplan-Meier survival curves, and equalities were compared using the logrank test. Cox proportional hazard analysis was performed to determine the independent associations between NAFLD at baseline and the development of low BMD.

\section{Ethics statement}

The present study was approved by the Research Ethics Committee of the First Affiliated Hospital, College of Medicine, Zhejiang University (IRB No.2018-980).

\section{Results}

\section{Description of the study population}

At baseline, there are a total of 1720 subjects, including 1064 subjects with normal BMD and 656 subjects with low BMD. Females comprised 26.3\%,mean age was $51.2 \pm 9.9$ years, and mean BMI was $24.1 \pm 3.2 \mathrm{~kg} / \mathrm{m}^{2}$. The baseline prevalence of NAFLD was $27.4 \%$ (Supplementary Table 1). We also conducted a cross-sectional analysis between normal BMD and low BMD groups. Compared to subjects with normal BMD at baseline, subjects with low BMD were more likely to be older, male, had lower BMI, and more likely to be current smokers, with a higher prevalence of NAFLD. For subjects with normal BMD, the metabolically glycometabolic laboratory parameters were healthier, although the difference was not statistically significant.

Then, we compared the baseline characteristics of 1064 participants between patients with and without incident low BMD during follow-up (Supplementary Table 2). 
Table 1 The associations between NAFLD and the development of low BMD.

\begin{tabular}{l} 
\\
\hline Total $(n=1064)$ \\
Non-NAFLD \\
NAFLD \\
Men $(n=751)$ \\
Non-NAFLD \\
NAFLD \\
Women $(n=313)$ \\
Non-NAFLD \\
NAFLD \\
Postmenopausal women $(n=144)$ \\
Non-NAFLD \\
NAFLD
\end{tabular}

\begin{tabular}{c}
\hline Unadjusted \\
\hline 1.00 (reference) \\
$1.62(1.32,2.00)$ \\
1.00 (reference) \\
1.91 (1.50, 2.44) \\
1.00 (reference) \\
1.35 (0.83, 2.20) \\
1.00 (reference) \\
1.44 (0.77, 2.70)
\end{tabular}

\begin{tabular}{c} 
Hazard ratios $(95 \% \mathrm{Cl})$ \\
\hline Model a \\
\hline 1.00 (reference) \\
$2.24(1.178,2.81)$ \\
1.00 (reference) \\
2.39 (1.85, 3.09) \\
1.00 (reference) \\
1.82 (1.09, 3.04) \\
1.00 (reference) \\
1.98 (0.99, 3.95)
\end{tabular}

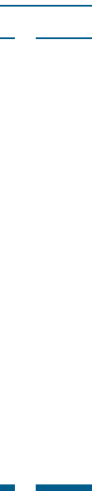

1.00 (reference)
$2.30(1.81,2.91)$
1.00 (reference)
$2.45(1.87,3.21)$
1.00 (reference)
1.74 (1.02, 2.98)
1.00 (reference)
2.13 (1.04, 4.33)

Multivariable adjusted Model a was adjusted for age, gender and BMI, except gender in the stratified analysis by gender; Model b: model 1 plus adjusted for serum Ca, smoking status (never, past and current smokers).

NAFLD, non-alcoholic fatty liver disease.

Men showed more unfavorable metabolic profiles than women. Participants with NAFLD revealed more unfavorable metabolic profiles, when compared with subjects without NAFLD (Supplementary Table 3).

\section{Baseline NAFLD and incident low BMD}

During the follow-up, 399 incident cases of low BMD were identified. Compared to subjects who developed low BMD, subjects with unchanged BMD were more likely to have higher BMI, SBP (systolic blood pressure), DBP (diastolic blood pressure), TG (triglyceride), VLDL (very low density lipoprotein) cholesterol and UA (uric acid), lower HDL (high-density lipoprotein) cholesterol, and decreased changes in TC (total cholesterol) and VLDL cholesterol (Supplementary Table 2). It is noteworthy that participants with NAFLD at baseline were more likely to develop low BMD during the follow-up period, compared to those without NAFLD (Table 1). The incidence of low BMD was 32.7 per 100 person-year of follow-up in participants without NAFLD, and this increased to 52.3 per 100 person-year in participants with baseline NAFLD.

Incident low BMD patients with NAFLD at baseline had higher BMI, SBP, DBP, serum TG, VLDL, UA and FPG levels, and lower HDL levels, while other metabolic parameters were comparable between the two groups (Supplementary Table 5).

\section{Risk of incident low BMD}

In the cohort before PSM, in the unadjusted model, the hazard ratio (HR) of incident low BMD in comparing NAFLD and non-NAFLD was 1.62 (1.32, 2.00). The cumulative incidence of low BMD was significantly higher in subjects with the presence of NAFLD compared to no NAFLD (Fig. 2, $P<0.01$ by log-rank test). HR was enhanced and remained statistically significant after adjusting for potential confounders. After adjusting variables in model 1 and model 2, the adjusted HR for incident low BMD comparing the NAFLD group vs the no NAFLD group was $2.24(1.18,2.81)$ and $2.30(1.81,2.91)$, respectively

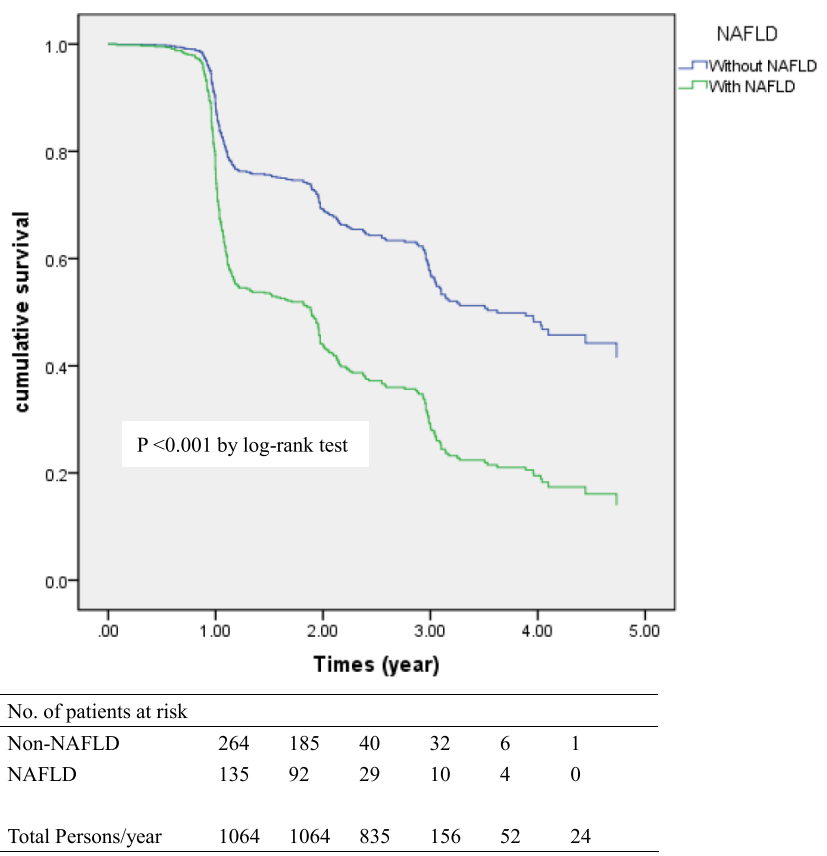

Figure 2

Follow-up normal BMD survival duration according to baseline NAFLD status. A full colour version of this figure is available at https://doi.org/10.1530/EJE-19-0699. 
Table 2 The associations between NAFLD and development of low BMD in the presence of obesity, defined as BMI $\geq 25 \mathrm{~kg} / \mathrm{m}^{2}$.

\begin{tabular}{|c|c|c|c|}
\hline & \multicolumn{3}{|c|}{ Hazard ratios $(95 \% \mathrm{Cl})$} \\
\hline & Unadjusted & Model $1 \mathrm{a}$ & Model $2 \mathrm{~b}$ \\
\hline \multicolumn{4}{|l|}{$\operatorname{Men}(n=751)$} \\
\hline \multicolumn{4}{|l|}{$\mathrm{BMI} \geq 25 \mathrm{~kg} / \mathrm{m}^{2}$} \\
\hline Non-NAFLD & 1.00 (reference) & 1.00 (reference) & 1.00 (reference) \\
\hline NAFLD & $4.41(2.86,6.80)$ & $4.51(2.92,6.97)$ & $5.26(3.31,8.36)$ \\
\hline \multicolumn{4}{|l|}{ Women $(n=313)$} \\
\hline \multicolumn{4}{|l|}{$\mathrm{BMI}<25 \mathrm{~kg} / \mathrm{m}^{2}$} \\
\hline Non-NAFLD & 1.00 (reference) & 1.00 (reference) & 1.00 (reference) \\
\hline NAFLD & $1.36(0.77,2.42)$ & $1.59(0.88,2.86)$ & $1.47(0.51,2.43)^{*}$ \\
\hline \multicolumn{4}{|l|}{$\mathrm{BMI} \geq 25 \mathrm{~kg} / \mathrm{m}^{2}$} \\
\hline Non-NAFLD & 1.00 (reference) & 1.00 (reference) & 1.00 (reference) \\
\hline NAFLD & $5.65(1.13,28.18)$ & $6.12(1.00,37.33)$ & $4.67(0.89,24.53)^{*}$ \\
\hline
\end{tabular}

Multivariable adjusted Model a was adjusted for age, gender and BMI, except gender in the stratified analysis by gender; Model b: Model 1 plus adjusted for serum Ca and smoking status. Obesity was defined according to the criteria for the Asian and Pacific regions (BMI $\geq 25 \mathrm{~kg} / \mathrm{m}^{2}$ ).

$\star P>0.05$

NAFLD, non-alcoholic fatty liver disease.

(Table 1). In subgroup analysis stratified by the presence of obesity, NAFLD was significantly associated with an increased risk of incident low BMD in both non-obese and obese individuals (Table 2). Obese women were more likely to develop low BMD. Due to a very few non-obese men included in this study, we did not specifically analyze relevant data of non-obese men.

Besides, we also performed univariate Cox regression (Supplementary Table 6). We found serum Ca was a protective factor, which was included in model 2. Serum phosphorus $(\mathrm{P})$, creatinine $(\mathrm{Cr})$ and glomerular filtration rate (GFR) did not show statistical significance.

\section{Propensity score-matched analysis}

In the former cross-sectional study about the association between low BMD and normal BMD, cases were more likely to be older, male, had lower BMI, and more likely to be current smokers, with a higher prevalence of NAFLD compared with normal. In order to further clarify the role of NAFLD in the development of NAFLD, we balanced the four factors (age, gender, obesity and smoking) between NAFLDs and non-NAFLDs. In this study, 256 pairs were matched using PSM. While in the cohort after PSM, NAFLD still remained an independent risk factor for the incidence of low BMD (HR 70.99, 95\% CI 16.42-307.04, $P<0.001$ ) (Supplementary Fig. 1 and Supplementary Table 4).

\section{Severity of NAFLD and incident low BMD}

In multivariate-adjusted models, an increase across baseline NAFLD categories based on APRI predicted an increase in the incidence of low BMD in a graded and dose-responsive manner (Fig. 3 and Table 3). The adjusted HRs for low BMD comparing NAFLD with low APRI and NAFLD with intermediate or high APRI vs no NAFLD were $2.16(1.71,2.73)$ and $3.08(1.86,5.10)$, respectively. The results were similar based on FIB- 4 .

\section{Discussion}

In this cohort study, we found NAFLD was associated with an increased risk of developing low BMD during longitudinal follow-up. Based on the non-invasive fibrosis

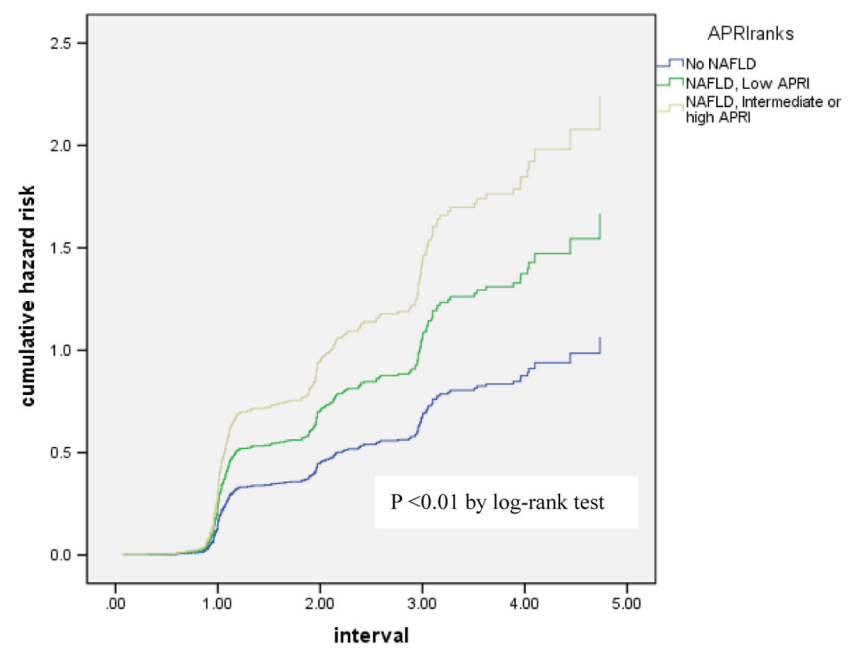

Figure 3

The association between NAFLD and its severity based on non-invasive fibrosis marker (APRI) and the development of low BMD. A full colour version of this figure is available at https://doi.org/10.1530/EJE-19-0699. 
Table 3 The associations between NAFLD and its severity based on non-invasive fibrosis markers and the development of low BMD.

\begin{tabular}{|c|c|c|c|}
\hline & \multicolumn{3}{|c|}{ Hazard ratios $(95 \% \mathrm{Cl})$} \\
\hline & Unadjusted & Model $1 \mathrm{a}$ & Model $2 \mathrm{~b}$ \\
\hline \multicolumn{4}{|l|}{ Based on APRI } \\
\hline Non-NAFLD & 1.00 (reference) & 1.00 (reference) & 1.00 (reference) \\
\hline NAFLD, Low APRI & $1.57(1.26,1.95)$ & $2.16(1.71,2.73)$ & $2.22(1.74,2.84)$ \\
\hline NAFLD, Intermediate or high APRI & $2.11(1.29,3.45)$ & $3.08(1.86,5.10)$ & $3.01(1.79,5.06)$ \\
\hline$P$ for trend & $<0.001$ & $<0.001$ & $<0.001$ \\
\hline \multicolumn{4}{|l|}{ Based on FIB-4 } \\
\hline Non-NAFLD & 1.00 (reference) & 1.00 (reference) & 1.00 (reference) \\
\hline NAFLD, Low FIB-4 & $1.58(1.23,2.02)$ & $2.24(1.72,2.92)$ & $2.37(1.81,3.12)$ \\
\hline NAFLD, Intermediate or high FIB-4 & $1.69(1.26,2.26)$ & $2.23(1.62,3.08)$ & $2.17(1.54,3.05)$ \\
\hline$P$ for trend & $<0.001$ & $<0.001$ & $<0.001$ \\
\hline
\end{tabular}

Multivariable adjusted Model a was adjusted for age, gender and BMI, except gender in the stratified analysis by gender; Model b: Model 1 plus adjusted for serum Ca and smoking status.

APRI, aspartate transaminase-to-platelet ratio index; FIB-4, fibrosis-4; NAFLD, non-alcoholic fatty liver disease.

markers, subjects with NAFLD and intermediate or high APRI and FIB-4 had the highest incidence of low BMD. Even with a low probability of hepatic fibrosis, NAFLD was still significantly associated with the development of low BMD. These associations persisted even after adjusting for possible confounders and PSM, suggesting an independent relationship exists between NAFLD and low BMD.

Several cross-sectional studies and only a few cohort studies have evaluated the association between NAFLD and low BMD, but the relationship remains controversial $(7,8,9,10,17,18)$. A recent meta-analysis of five crosssectional studies revealed that there was no significant difference in BMD between patients with NAFLD and controls (18). The other meta-analysis also showed NAFLD was associated with self-reported osteoporotic fracture, but not with low BMD (19). The updated metaanalysis based on children and adolescents found that the presence and severity of NAFLD were significantly associated with reduced whole-body BMD Z scores (10). Recently, a cohort study of 21590 Chinese health checkup examinees demonstrated that NAFLD might increase the risk of developing new-onset osteoporosis (20). In our study, NAFLD was independently associated with an increased risk for developing low BMD in both men and women. Even in non-obese subjects and after adjusting potential factor, positive association between NAFLD and incident low BMD was still observed. Indeed, NAFLD, even in non-obese individuals, is associated with IR, impaired glucose tolerance, and metabolic syndrome, which are also risk factors for low $\operatorname{BMD}(21,22)$.

With regard to NAFLD severity, we used two noninvasive indices. In our study, increasing severity of NAFLD from low to intermediate or high fibrosis marker at baseline was associated with higher incidence of low BMD in a graded manner. Even NAFLD with low fibrosis marker was also associated with a higher incidence of low BMD, compared with no NAFLD. However, due to limited participants with a high probability of fibrosis, we did not have a separate category for a more severe form of NAFLD. A recent study assessed the association between liver fibrosis via TE and BMD in NAFLDs (11). They found significant liver fibrosis was independently correlated with low BMD at the femur neck $(\beta= \pm 0.18)$ and total hip $(\beta= \pm 0.21)$ after adjustment for several confounding variables, such as age, sex, BMI, and indicators of glucose and lipid metabolism among NAFLDs. Different from their study, we used two simple and accessible biomarkers, APRI and FIB-4. One of the biggest advantages is convenient calculation, as they are based on widely available and simple parameters (age, transaminases, platelets and so on) and more attractive to general practitioners. Furthermore, APRI and FIB- 4 for diagnosing advanced fibrosis reported summary AUROCS of 0.77 and 0.84 respectively (23). It is obvious that APRI and FIB-4 have lower accurate diagnostic performance than TE (23). Besides, it is also reported that when using FIB-4, there was a significant segment of patients (around $30 \%)$ who fall in the intermediate-risk category and cannot be correctly classified (24).

In our study, the association between NAFLD and development of low BMD was similarly observed in both men and women, whereas the association between NAFLD and incident low BMD tended to be stronger in men than women. NAFLD, a sexual dimorphic disease, is more common in men and postmenopausal women, and low BMD is more common in postmenopausal women (21, 25). Similarly, in our study, men were found to be more likely to have NAFLD, while postmenopausal women and 
obese subjects were more likely to develop low BMD. In addition, our study suggested a stronger association of obesity with the risk of low BMD in women than in men. Accounting for the differences between men and women, body fat deposition and sex hormone levels could be potential explanations. Estrogen deficiency is known to be the major cause of bone loss in postmenopausal women $(26,27)$. Estrogen could regulate osteoclast activity through estrogen receptor and thereby acts to conserve bone mass by inhibiting bone resorption $(28,29)$. NAFLD and the effect of estrogen deficiency in women might act additively or synergistically incontributing to the development of low BMD. Further studies are needed to fully explain the role of NAFLD in the development of low BMD while considering the existence of different effects by gender.

The present study has several potential limitations. First, NAFLD was diagnosed based on ultrasound results, which may have underestimated the incidence of NAFLD. So we also used other two non-invasive markers to assess the severity of NAFLD to make up for deficiencies. Secondly, due to a very few non-obese men included in this study, we did not specifically analyze the data of non-obese men, which may influence the result. We now continue to collect related data and expect to enlarge the sample size. Thirdly, we acknowledged that lack of concrete data on BMD and T-scores is the main limitation of this article. Due to some objective factors, we were unable to obtain this batch of clinical data. Furthermore, we could not do subgroup comparison of BMD values measured by DXA at different skeletal sites (lumbar spine and total hip) between the NAFLDs and non-NAFLDs. Finally, the lack of any information about the use of drugs potentially influencing BMD (e.g., use of vitamin D supplements, anti-osteoporotic agents or steroids), could have influenced the result.

Unlike the previous cohort study (20), we assessed the impact of NAFLD and its severity on the development of low BMD. And we also performed PSM to reduce selection bias, and did subgroup analysis according to the presence of obesity in both men and women, separately.

In conclusion, the present study revealed that NAFLD and non-invasive fibrosis markers were independently associated with an increased incidence of low BMD. In addition, subjects with NAFLD may need to be screened for low BMD, especially for obese women and men. Considering the increasing rate of osteoporosis worldwide and lack of approved drug therapy for osteoporosis, these present findings may shed light on potential preventive and therapeutic interventions to treat osteoporosis.
Furthermore, these findings also support the hypothesis that NAFLD may contribute to the pathogenesis of low BMD. This research question, together with a more in-depth investigation of gender differences, needs to be addressed in future studies.

\section{Supplementary materials}

This is linked to the online version of the paper at https://doi.org/10.1530/ EJE-19-0699.

\section{Declarations of interest}

The authors declare that there is no conflict of interest that could be perceived as prejudicing the impartiality of this study.

\section{Funding}

Supported by National Key R\&D Program of China (2017YFC0908900) and National Natural Science Foundation of China (No. 81770586). The funding source had no involvement in the study.

\section{Ethical statement}

Consent has been obtained from each patient or subject after full explanation of the purpose and nature of all procedures used.

\section{Author contribution statement}

Chen $\mathrm{W}$ and Yu C conceived and designed the study. Shen Z, Cen L, Chen X, Pan J, Li Y collected the data. Shen Z, Cen L analyzed the data. Shen Z, Cen $L$ wrote the paper. Final approval of the paper was by Shen $Z$, Cen $L$, Chen $X$, Pan J, Li Y, Chen W and Yu C. All authors approved the final version of the manuscript. Guarantors of the article are Chen W and Yu C.

\section{Acknowledgements}

The authors are grateful to Stefan Munker for his assistance with language correction and guidance.

\section{References}

1 Marshall D, Johnell O \& Wedel H. Meta-analysis of how well measures of bone mineral density predict occurrence of osteoporotic fractures. BMJ 1996312 1254-1259. (https://doi.org/10.1136/ bmj.312.7041.1254)

2 Sanchez-Riera L, Carnahan E, Vos T, Veerman L, Norman R, Lim SS, Hoy D, Smith E, Wilson N, Nolla JM et al. The global burden attributable to low bone mineral density. Annals of the Rheumatic Diseases 201473 1635-1645. (https://doi.org/10.1136/ annrheumdis-2013-204320)

3 Fan JG, Kim SU \& Wong VW. New trends on obesity and NAFLD in Asia. Journal of Hepatology 201767 862-873. (https://doi. org/10.1016/j.jhep.2017.06.003)

4 Farrell GC, Wong VW \& Chitturi S. NAFLD in Asia - as common and important as in the West. Nature Reviews: Gastroenterology and Hepatology 201310 307-318. (https://doi.org/10.1038/ nrgastro.2013.34)

5 VanWagner LB \& Rinella ME. Extrahepatic manifestations of nonalcoholic fatty liver disease. Current Hepatology Reports 201615 75-85. (https://doi.org/10.1007/s11901-016-0295-9) 
6 DiGirolamo DJ, Clemens TL \& Kousteni S. The skeleton as an endocrine organ. Nature Reviews: Rheumatology 20128 674-683. (https://doi.org/10.1038/nrrheum.2012.157)

7 Pardee PE, Dunn W \& Schwimmer JB. Non-alcoholic fatty liver disease is associated with low bone mineral density in obese children. Alimentary Pharmacology and Therapeutics 201235 248-254. (https://doi.org/10.1111/j.1365-2036.2011.04924.x)

8 Lee DY, Park JK, Hur KY \& Um SH. Association between nonalcoholic fatty liver disease and bone mineral density in postmenopausal women. Climacteric 201821 498-501. (https://doi.org/10.1080/1369 7137.2018.1481380)

9 Umehara T. Nonalcoholic fatty liver disease with elevated alanine aminotransferase levels is negatively associated with bone mineral density: cross-sectional study in U.S. adults. PLOS ONE 201813 e0197900. (https://doi.org/10.1371/journal.pone.0197900)

10 Mantovani A, Gatti D, Zoppini G, Lippi G, Bonora E, Byrne CD, Nobili V \& Targher G. Association between nonalcoholic fatty liver disease and reduced bone mineral density in children: a meta-analysis Hepatology 201970 812-823. (https://doi.org/10.1002/hep.30538)

11 Kim G, Kim KJ, Rhee Y \& Lim SK. Significant liver fibrosis assessed using liver transient elastography is independently associated with low bone mineral density in patients with non-alcoholic fatty liver disease. PLOS ONE 201712 e0182202. (https://doi.org/10.1371/ journal.pone.0182202)

12 Targher G, Lonardo A. \& Rossini M. Nonalcoholic fatty liver disease and decreased bone mineral density: is there a link? Journal of Endocrinological Investigation 201538 817-825. (https://doi. org/10.1007/s40618-015-0315-6)

13 Kanis JA, Melton 3rd LJ, Christiansen C, Johnston CC \& Khaltaev N. The diagnosis of osteoporosis. Journal of Bone and Mineral Research 19949 1137-1141. (https://doi.org/10.1002/jbmr.5650090802)

14 Fan JG \& Farrell GC. Epidemiology of non-alcoholic fatty liver disease in China. Journal of Hepatology 200950 204-210. (https://doi. org/10.1016/j.jhep.2008.10.010)

15 Shah AG, Lydecker A, Murray K, Tetri BN, Contos MJ, Sanyal AJ \& Nash Clinical Research Network. Comparison of noninvasive markers of fibrosis in patients with nonalcoholic fatty liver disease. Clinical Gastroenterology and Hepatology 20097 1104-1112. (https:// doi.org/10.1016/j.cgh.2009.05.033)

16 Wai CT, Greenson JK, Fontana RJ, Kalbfleisch JD, Marrero JA, Conjeevaram HS \& Lok AS. A simple noninvasive index can predict both significant fibrosis and cirrhosis in patients with chronic hepatitis C. Hepatology 200338 518-526. (https://doi.org/10.1053/ jhep.2003.50346)

17 Wang Y, Wen G, Zhou R, Zhong W, Lu S, Hu C \& Chai Y. Association of nonalcoholic fatty liver disease with osteoporotic fractures: a cross-sectional retrospective study of Chinese individuals. Frontiers in Endocrinology 20189 408. (https://doi.org/10.3389/ fendo.2018.00408)

18 Upala S, Jaruvongvanich V, Wijarnpreecha K \& Sanguankeo A. Nonalcoholic fatty liver disease and osteoporosis: a systematic review and metaanalysis. Journal of Bone and Mineral Metabolism 201735 685-693. (https://doi.org/10.1007/s00774-016-0807-2)
19 Mantovani A, Dauriz M, Gatti D, Viapiana O, Zoppini G, Lippi G, Byrne CD, Bonnet F, Bonora E \& Targher G. Systematic review with meta-analysis: non-alcoholic fatty liver disease is associated with a history of osteoporotic fractures but not with low bone mineral density. Alimentary Pharmacology and Therapeutics 201949 375-388. (https://doi.org/10.1111/apt.15087)

20 Chen HJ, Yang HY, Hsueh KC, Shen CC, Chen RY, Yu HC \& Wang TL. Increased risk of osteoporosis in patients with nonalcoholic fatty liver disease: a population-based retrospective cohort study. Medicine 201897 e12835. (https://doi.org/10.1097/MD.0000000000012835)

21 Filip R, Radzki RP \& Bienko M. Novel insights into the relationship between nonalcoholic fatty liver disease and osteoporosis. Clinical Interventions in Aging 201813 1879-1891. (https://doi.org/10.2147/ CIA.S170533)

22 Yang Y, Wang B, Fei Q, Meng Q, Li D, Tang H, Li J \& Su N. Validation of an osteoporosis self-assessment tool to identify primary osteoporosis and new osteoporotic vertebral fractures in postmenopausal Chinese women in Beijing. BMC Musculoskeletal Disorders 201314 271. (https://doi.org/10.1186/1471-2474-14-271)

23 Xiao G, Zhu S, Xiao X, Yan L, Yang J \& Wu G. Comparison of laboratory tests, ultrasound, or magnetic resonance elastography to detect fibrosis in patients with nonalcoholic fatty liver disease: a meta-analysis. Hepatology 201766 1486-1501. (https://doi. org/10.1002/hep.29302)

24 Alexander M, Loomis AK, Fairburn-Beech J, van der Lei J, DuarteSalles T, Prieto-Alhambra D, Ansell D, Pasqua A, Lapi F, Rijnbeek P et al. Real-world data reveal a diagnostic gap in non-alcoholic fatty liver disease. BMC Medicine 201816 130. (https://doi.org/10.1186/ s12916-018-1103-x)

25 Ballestri S, Nascimbeni F, Baldelli E, Marrazzo A, Romagnoli D \& Lonardo A. NAFLD as a sexual dimorphic disease: role of gender and reproductive status in the development and progression of nonalcoholic fatty liver disease and inherent cardiovascular risk. Advances in Therapy 201734 1291-1326. (https://doi.org/10.1007/ s12325-017-0556-1)

26 Moon SS, Lee YS \& Kim SW. Association of nonalcoholic fatty liver disease with low bone mass in postmenopausal women. Endocrine 201242 423-429. (https://doi.org/10.1007/s12020-012-9639-6)

27 Xia MF, Lin HD, Yan HM, Bian H, Chang XX, Zhang LS, He WY $\&$ Gao X. The association of liver fat content and serum alanine aminotransferase with bone mineral density in middle-aged and elderly Chinese men and postmenopausal women. Journal of Translational Medicine 201614 11. (https://doi.org/10.1186/s12967016-0766-3)

28 Melville KM, Kelly NH, Khan SA, Schimenti JC, Ross FP, Main RP \& van der Meulen MC. Female mice lacking estrogen receptor-alpha in osteoblasts have compromised bone mass and strength. Journal of Bone and Mineral Research 201429 370-379. (https://doi.org/10.1002/ jbmr.2082)

29 Kodama I, Niida S, Sanada M, Yoshiko Y, Tsuda M, Maeda N \& Ohama K. Estrogen regulates the production of VEGF for osteoclast formation and activity in op/op mice. Journal of Bone and Mineral Research 200419 200-206. (https://doi.org/10.1359/JBMR.0301229)

Received 4 September 2019

Revised version received 20 November 2019

Accepted 26 November 2019 